\title{
Network Analysis, and Experimental Validation to Uncover the Mechanism of the Four Compounds in Artemisia annua (Qing Hao) Antimalarial
}

\section{Yuping Zhao}

China Academy of Chinese Medical Sciences

\section{Xiaobo Zhang}

China Academy of Chinese Medical Sciences

Haiyu Xu

China Academy of Chinese Medical Sciences

\section{Ling Wang}

China Academy of Chinese Medical Sciences

Luqi Huang ( $\nabla$ huangluqi01@126.com )

China Academy of Chinese Medical Sciences

\section{Research}

Keywords: Artemisia annua, antimalaria, bioinformatics, interactive mechanism, synergistic effect

Posted Date: July 7th, 2020

DOl: https://doi.org/10.21203/rs.3.rs-19055/v2

License: (c) (i) This work is licensed under a Creative Commons Attribution 4.0 International License. Read Full License 


\section{Abstract}

Background: Artemisinin is widely used to anti malaria, but the antimalaria mechanism and coordinative interaction of Artemisinin, Scopoletin, Arteannuin B and Artemisic acid are not clear.

Methods: Based on the existing of antimalarial drugs, the antimalaria targets of Artemisinin, Scopoletin, Arteannuin B and Artemisic acid were explored by molecular docking with the similarity theory of chemical structure, and then the antimalaria mechanism of Scopoletin and its coordinative antimalaria interaction of the mixture with the three other ingredients.

Results: Then through using the text information excavation's method, the relevance proteins of antimalaria effect of Artemisinin were IL-6, ACHE, PC3, IPOB, CYC, TNF-a, UGT1A9, CASP3, XDH, IL-1 3 , VEGF, CAT, CREB, AMPK, UGT1A6, ADR, MAPK, COX2, LB24AB and CYP450. Meanwhile, the relevance proteins of Scopoletin were TNF-a, PI3K, IL-8, IL- 6, VEGF, IL-1ß, MAPK, CD4, SP2, CTNNB, CASP3, PR01400, IgE, IL-4, ICAM1, p38, STAT3, TLR4 and API4. But Arteannuin B and Artemisic acid had a little relevance with the above proteins. The interaction characteristic between TNF- $\alpha$ and Artemisia annua was the effect of the mixture of Artemisinin, Scopoletin, Arteannuin B and Artemisic acid was greater than Artemisinin, and the synergistic effect of the four elements was found in the progress of antimalaria.

Conclusion: Antimalarial target of Artemisia annua ingredients was explored with data mining methods, and the antimalarial effect of Scopoletin may be related to TNF. Combined application of the four elements could achieve the same antimalarial effect and reduce the clinical usage of Artemisinin and Scopoletin.

\section{Background}

Malaria is a major threat to human life. Together with AIDS and cancer, it is listed by the World Health Organization as one of the world's three major deadly diseases. Before the emergence and promotion of artemisinin, about 400 million people worldwide were infected by and at least 1 million people died from malaria every year. Morbidity and mortality of malaria are especially high in sub-Saharan Africa. According to WHO data in 2016, 2.7 billion dollars was spent on malaria control and elimination globally by governments and international organizations [1]. Antimalarial drugs mainly include quinolines (such as chloroquine, mefloquine, quinine, etc.) and antifolates (such as pyrimethamine, sulfadoxine, etc.). The use of these drugs has effectively controlled the global spread of malaria. However, Plasmodium falciparum then developed resistance to almost all antimalarial drugs [2]. China was once one of the major countries affected by malaria, and made tremendous efforts to eliminate the disease in the country [3]. Unique chemical composition in traditional Chinese materia medica has significant biological activity in major diseases. Chinese medicine with clear active ingredients is rare, while Artemisia annua (Qing $\mathrm{HaO}$ ) is a typical chemical composition obtained by modern scientifically verified traditional Chinese materia medica. The victory was finally achieved, in 2014, malaria patients were controlled to 56 individuals [4]. In 1990s, artemisinin was widely used in Thailand and other countries in Southeast Asia. 
Then it was widely adopted in Africa and the Americas [5]. However, neither artemisinin monotherapy nor artemisinin-based combination therapy showed any efficacy and delayed parasite clearance often confused the clinicians, as same as other antimalarial drugs [6-8]. Plasmodium falciparum has developed resistance to artemisinin in the Greater Mekong region, including Cambodia, Laos, Myanmar, Thailand and Vietnam. The World Health Organization's 2011 global plan strived to tackle artemisinin resistance, due to the artemisinin partner drug [9]. The reason might mainly from the artemisinin partner drug [10]. Artemisinin if combined with another drug with low drug resistance, would not delay parasite clearance. It remains completely possible to rely on artemisinin and its new partner drugs to stop the prevalence of malaria [11]. The drug with low drug resistance must be a new one, as existing antimalarials drugs have developed strong resistance. Scopoletin, Arteannuin B and Artemisic acid were considered because of their chemical structure are more similar to Artemisinin. Compounds that selectively act on two or more targets of interest in theory would perform more pharmacological action than single-target agents [12]. 'Polypharmacology' is known as a new terminology that followed by drug finder [13]. This topic uses the method of network pharmacology to carry out text mining and target prediction by recording the four components of artemisinin, artemisinin, artemisinic acid and sorghum lactone, which may have antimalarial effects in Artemisia annua L. Molecular docking is an essential procedure to verify network pharmacology in structural molecular biology and computer-assisted drug design. Molecular docking could be used to perform virtual screening on chemical compounds, rank the results, and propose structural hypotheses on how the ligands inhibit the target, which is invaluable in lead optimization [14].

\section{Materials And Methods}

\section{Reagents and materials}

1-ethyl-3-(3-dimetylaminopropyl)-carbodiimide hydrochloride (EDC) was obtained from Shanghai Beinuo Biotechnology Co. Ltd. (Shanghai, China), N-hydroxysuccinimide (NHS) was from Shanghai Dibai Biotechnology Co. Ltd. (Shanghai, China), Sodium acetate and dimethyl sulfoxide (DMSO) were from Beijing Chemical Plant (Beijing, China), Ethanolamine hydrochloride was purchased from Shanghai Sanying Chemical Reagent Co. Ltd. (Shanghai, China), the protein TNF-a was provided by RD Biosciences (America), Artemisinin, Scopoletin, Arteannuin B and Artemisic acid were provided by Chengdu Ruifensi Biological Technology Co., Ltd. (Chengdu, China), Dulbecco's phosphate buffered saline PBS buffer (PH4.7) were freshly prepared.

\section{Plasma sample preparation}

Weigh accurately $40 \mathrm{mg}$ of EDC and $10 \mathrm{mg}$ of $\mathrm{NHS}$, and prepare $1 \mathrm{~mL}$ solution with distilled water. Inject the above solution within 5 minutes into two channels thoroughly rinsed by PBS buffer.

$50 \mu \mathrm{g}$ of TNF-a protein was dissolved in $100 \mu \mathrm{L}$ of PBS, and $10 \mu \mathrm{L}$ of the above solution was taken in three portions and diluted with sodium acetate solutions with $\mathrm{pH}$ values of $5.5,6.0$, and 6.5 , respectively, 
and the final concentration was $50 \mu \mathrm{g} / \mathrm{ml}$. Reduce the flow rate to $20 \mu \mathrm{L} / \mathrm{min}$ and rinse the left channel for 10 minutes to determine the optimal $\mathrm{pH}$ of sodium acetate.

After determination of the optimal $\mathrm{pH}$ value, $1 \mathrm{M}$ ethanolamine hydrochloride was injected into the two channels for 10 minutes to complete the sample fixation. The four chemical composition Scopoletin (A), Artemisinin (B), Artemisic acid (C)and Arteannuin $B(D)$ were divided into 12 groups according to the combination rule of $A, B, C, D, A B, A C, A D, A B C, A B D, A C D, B C D$, and $A B C D$, and each group has 6 concentration levels. The control group was PBS buffer at $\mathrm{pH} 7.4$.

Accurately weigh $19.2 \mathrm{mg}$ of scutellaria lactone, $28.2 \mathrm{mg}$ of artemisinin, $24.8 \mathrm{mg}$ of artemisinin 2 and $23.4 \mathrm{mg}$ of artemisinin, and dissolve them in $1 \mathrm{~mL}$ DMSO (dimethyl sulfoxide), respectively. The DMSO solution in each group was gradient diluted with PBS to a final concentration of $200 \mu \mathrm{M}, 66.7 \mu \mathrm{M}, 22.2$ $\mu \mathrm{M}, 7.41 \mu \mathrm{M}, 2.47 \mu \mathrm{M}$.

\section{Targets fishing}

Known therapeutic targets for the treatment of malaria were obtained from the DrugBank database (http://www.drugbank.ca/, version, 4.3) [15]. The prediction of drug targets based on ligand structural features mainly includes chemical similarity search and reverse pharmacophore search. The theoretical basis of chemical similarity search is that small molecular compounds with similar structural or physicochemical properties can act on targets with the same or similar properties "Antimalaria" was selected as the key word, and the drug-target interactions whose drugs are approved by the Food and Drug Administration, USA (FDA) for treating menstrual disorders. All target gene/ protein identifiers (IDs) were converted into the correspondinggene symbol/UniProtKB-Swiss-Prot IDto facilitate further data analyses. After removing redundant entries, 25 target genes corresponding to 15 known antimalarial drugs were retrieved.

\section{Protein-protein interaction (PPI) data}

PPI data were imported from the following PPI databases, including Human Annotated and Predicted Protein Interaction Database (HAPPI, http://bio.informatics. iupui.edu/HAPPI/, Version 31.2) [16]. Based on the PPI network database, an interaction network of Artemisia annua candidate target groups and known antimalarial drug target groups was constructed, and the distribution of target nodes in metabolic pathways and the corresponding diseases were revealed. As a result, a direct interaction network of key nodes was then established, and divided into different modules according to the functions of the nodes. According to the malaria pathway (ko05144: Malaria) in KEGG, molecules closely related to the malaria pathway were selected as candidates to be verified from the key nodes.

\section{Network construction and topological analysis}

Compound-target (C-T), target-pathway (T-P), and target-disease (T-D) networks of malaria were constructed using the Cytoscape 3.2 software (https://cytoscape. org/download.html), a general bioinformatics software package for data integration and visualization of biological network (Bindea et 
al., 2009; Smoot et al., 2011). An interaction network of Artemisia annua candidate target genes with known antimalarial drug target genes was established, consisting of 85 nodes and 298 pairs of interactions. The topological characteristic value of each node was calculated in the network, and the median of the topological characteristic value was used as the card value. A total of 32 key nodes were screened. A direct interaction network of key nodes was established, and processed according to the node functions. The module was divided, and the malaria pathway in Kyoto Encyclopediaof Genesand Genomes (KEGG) (ko05144: Malaria) was compared, and molecules closely related to the malaria pathway were selected as candidates to be verified from the key nodes.

\section{Molecular docking.}

The molecular structures of CDK4, NFKB1, PIK3CG, MAPK1, TNF and ITGB2 protein targets (protein species is human) were searched in the database UniProt (http://www.uniprot.org/). The structures of scopolamine and artemisinic acid are downloaded from the PubChem database (https://pubchem.ncbi.nIm.nih.gov). Chemical composition and protein structure were dehydrated and hydrotreated. Molecular docking and figures were generated Discovery Studio (DS2.5) Visualizer software.

\section{Probe Kd determination}

Weigh accurately $40 \mathrm{mg}$ of EDC and $10 \mathrm{mg}$ of NHS, and prepare $1 \mathrm{~mL}$ solution with distilled water. Inject the above solution within 5 minutes into two channels thoroughly rinsed by PBS buffer. Precisely weigh $19.2 \mathrm{mg}$ of scopolamine, $28.2 \mathrm{mg}$ of artemisinin, $24.8 \mathrm{mg}$ of artemisinin, and $23.4 \mathrm{mg}$ of artemisinic acid in $1 \mathrm{~mL}$ DMSO (dimethyl sulfoxide) and mix well. The protein TNF-a immobilized on Grafted Sensor Chips. The compound monomers and combinations are divided into 12 groups (Table 1). Each group of samples was injected from low to high concentration, and a control group (PBS) was set at each concentration. Regression analysis when concentration curves are separated and approaching equilibrium. The dissociation constant $(\mathrm{Kd})$ and its maximum value (Bmax) was then calculated by fitting the titration curve to the single-site saturation binding equation $[Y=B m a x * X /(K d+X)]$ using the GraphPad Prism software (Graphpad software Incorporated, La Jolla, CA, USA).

Table 1 The compound monomers and combinations 


\begin{tabular}{ll}
\hline Group & compounds \\
\hline A & Scopoletin \\
B & Artemisinin \\
\hline C & Artemisic acid \\
\hline D & Arteannuin B \\
\hline AB & Scopoletin;Artemisinin \\
\hline AC & Scopoletin; Artemisic acid \\
\hline AD & Scopoletin; Arteannuin B \\
\hline ABC & Scopoletin; Artemisinin ;Artemisic acid \\
\hline ABD & Scopoletin; Artemisinin; Arteannuin B \\
\hline ACD & Scopoletin; Artemisic acid; Arteannuin B \\
\hline BCD & Artemisinin ;Artemisic acid; Arteannuin B \\
\hline
\end{tabular}

\section{Results}

\section{Compound-target network construction}

There were 162 diseases related to four chemical components in Artemisia annua found. Through artificial noise reduction, the top 5 diseases ranked by frequency of occurrence were extracted as malaria (50), cerebral malaria (7), falciparum malaria (4), visceral leishmaniasis (2), and systemic lupus erythematosus (1) (Figure 1).

There were 15 known antimalarial drugs retrieved from DrugBank database(Table 2). There are 93 targets with structures similar to scopolide and 15 known antimalarial drugs (Similar score more than 0.7). Artemisinic acid had 32 targets with structures similar to 15 known antimalarial drugs (Similar score than $0.7)$.

\section{Clustering analysis}

The key node interaction network was divided into three functional modules. The first functional module mainly involved immune-related pathways, the second mainly involves multiple infectious diseases, and the third was related to drug metabolism and tumor pathways. KEGG's malaria pathway (ko05144:

Malaria) comparison was shown in Figure 2. The four key nodes were involved in four pathways: T cell receptor signaling pathway, Toll-like receptor signaling pathway, TNF signaling pathway, Natural killer cell mediated cytotoxicity are all in the malaria pathway Important links, so the joint nodes involved in these four pathways can be regarded as a candidate to be verified. 
Table 2 Known antimalarial drugs

\begin{tabular}{|c|c|}
\hline Drug ID & Name \\
\hline DB00254 & Doxycycline \\
\hline DB00908 & Quinidine \\
\hline DB00358 & Mefloquine \\
\hline DB01611 & Hydroxychloroquine \\
\hline DB00608 & Chloroquine \\
\hline DB00613 & Amodiaquine \\
\hline DB01103 & Quinacrine \\
\hline DB01087 & Primaquine \\
\hline DB00468 & Quinine \\
\hline DB04877 & Voacamine \\
\hline DB00440 & Trimethoprim \\
\hline DB01218 & Halofantrine \\
\hline DB00205 & Pyrimethamine \\
\hline DB01131 & Proguanil \\
\hline DB01117 & Atovaquone \\
\hline
\end{tabular}

\section{Molecular docking}

Scopolide had suitable docking sites with TNF, NFKB1, and PIK3CG, and the docking scores were 77.9576, 57.8491, and 50.2248 respectively, while no suitable docking site with CDK4, MAPK1. There had no suitable docking site for Artemisinic acid with ITGB2 (Table 3, Figure 3).

Table 3 Docking score of Scopolide and artemisinic acid with the targets 


\begin{tabular}{ccc}
\hline Compounds & Tagets & Docking Score \\
\hline Scopolide & CDK4 & - \\
Scopolide & NFKB1 & 57.8491 \\
Scopolide & PIK3CG & 50.2248 \\
& & \\
Scopolide & MAPK1 & - \\
Scopolide & TNF & 77.9576 \\
& & \\
artemisinic acid & ITGB2 & - \\
\hline
\end{tabular}

\section{Experimental validation of key targets}

According to the binding curve of the immobilized protein, the highest binding efficiency of sodium acetate solution at $\mathrm{pH} 5.5$ is $110 \mu \mathrm{RIU} / \mathrm{s}$, but the binding is unstable. The lowest binding efficiency at pH 6.5 is $68 \mu \mathrm{RIU} / \mathrm{s}$. At pH 6.0, the binding efficiency is better and the comparison of binding It is stable at $108 \mu \mathrm{RIU} / \mathrm{s}$, so it is best to use sodium acetate solution with $\mathrm{pH} 6.0$ for protein fixation.

TNF had good binding with scopolide, suggesting that the antimalarial effect of scopolide may be related to TNF. The binding of TNF to artemisinin, artemisinin B, and artemisinic acid was weak, but the combination of the 4 components of artemisinin, artemisinin B, artemisinic acid, and stigmalactone had good binding to TNF, suggesting the combined application of 4 ingredients may achieve antimalarial effects by acting on TNF (Table 4, Figure 4).

Table 4 Equilibrium dissociation constant $\mathrm{KD}$ for each group 


\begin{tabular}{cccc}
\hline Group & KD(M) & Est. error & Bmax Signal (uRIU) \\
\hline A & $4.05 \times 10^{-6}$ & $2.00 \times 10^{-6}$ & 42.0 \\
B & $2.01 \times 10^{-4}$ & $1.45 \times 10^{-5}$ & 38.7 \\
\hline C & $8.91 \times 10^{-5}$ & $4.07 \times 10^{-7}$ & 72.3 \\
\hline D & $2.93 \times 10^{18}$ & $1.39 \times 10^{-7}$ & $2.78 \times 10^{17}$ \\
\hline AB & $1.35 \times 10^{-3}$ & $8.08 \times 10^{-6}$ & 46.7 \\
\hline AC & $1.25 \times 10^{-4}$ & $3.97 \times 10^{-6}$ & 33.7 \\
\hline AD & $3.29 \times 10^{-4}$ & $1.72 \times 10^{-6}$ & 15.4 \\
\hline ABD & $1.20 \times 10^{-4}$ & $1.61 \times 10^{-6}$ & 12.7 \\
\hline ABC & $2.69 \times 10^{-4}$ & $8.38 \times 10^{-7}$ & 25.8 \\
\hline ACD & $1.59 \times 10^{20}$ & $4.91 \times 10^{-7}$ & $8.36 \times 10^{18}$ \\
\hline BCD & $2.80 \times 10^{-5}$ & $1.78 \times 10^{-5}$ & 10.1 \\
\hline ABCD & $2.17 \times 10^{-5}$ & $5.04 \times 10^{-5}$ & 12.5 \\
\hline & & & \\
\hline
\end{tabular}

\section{Discussion}

The sesquiterpene compounds represented by artemisinin are the most active field in Artemisia annua. At present, nearly 61 sesquiterpenoids, mainly artemisinin compounds, have been identified from Artemisia annua, including artemisinic acid, artemisinol, artemisinin ether and artemisinin. Artemisinin is a sesquiterpene lactone containing an endoperoxy bridge structure and is the main component of antimalarial. Research data showed that artemisinin and artemisinin can be converted to artemisinin in the original plants $[17,18]$. The biosynthetic routes of artemisinin can be summarized as 9 total synthetic routes and 5 semi-synthetic routes. The synthesizing method of artemisinin has a long process, high cost, and low total output, maximum to $10 \%$. Artemisinic acid is one of the main components of sesquiterpenes in Artemisia annua plants and an important precursor of artemisinin synthesis. Tu Yu's study showed that the young plants of Artemisia annua contained a large amount of artemisinic acid but was short of artemisinin. It is speculated that sesquiterpenoids such as artemisinin were converted from artemisinic acid. Researchers like Levesque $\mathrm{F}$ and others used synthetic biology to successfully produce artemisinin using genetically engineered yeast, and synthesized artemisinin [19]. Scopolide has strong water solubility and stability to artemisinin, etc., and has pharmacological activity that reflects the efficacy of traditional artemisinin. Studies have shown that scopolide has a certain antimalarial effect, and it has certain effects with artemisinin synergy. With the rapid development of chemical genomes and pharmacological technologies, a large number of potential targets and massive biological activity data have emerged. However, with the accumulation of complex data, simple analysis methods can no longer satisfy the analytical needs of high-throughput and large-scale data [20]. The rapid development of 
chemical informatics has just met the requirements of big data processing and information extraction tasks that urgently needs to be solved in chemical genomics. Chemical informatics mainly studies how to properly select the diversity of compound libraries, how to describe drug molecular characteristics, how to measure the differences between different molecules, how to identify drug-like molecules, molecular structure and biological performance relationships, and how to develop corresponding computer software and hardware. This includes research tasks and content of chemometrics and computational chemistry [21]. An important application of the chemoinformatics method in the post-genomic era is to predict the potential targets of small molecule compounds based on existing biological and chemical information, and to explain their mechanism of action to accelerate the development of drugs. The prediction of drug targets is of great significance to the evaluation of early drug molecules and the new use of old drugs, however, due to the limitations of throughput, accuracy and cost, it is difficult to widely apply experimental methods. As a quick and low-cost method, the development of computer-aided target prediction algorithms is receiving more and more attention. According to different research strategies, prediction of drug targets based on chemoinformatics can be divided into three categories: prediction based on ligand characteristics, prediction based on protein structural characteristics, and prediction based on data mining methods $[22,23]$. According to the target prediction, it is found that the potential targets of scopolide are CDK4, NFKB1, PIK3CG, MAPK1, TNF, and the potential target of artemisinic acid is ITGB2. Cyclin-dependent kinase (CDK) is a type of serine / threonine (Thr) kinase, which is an important signal transduction molecule in cell, and CDK-cyclin formed by cyclin complex, and involved in cell growth, proliferation, dormancy, or entering apoptosis. During the cell cycle, cyclins are periodically and continuously expressed and degraded, and are respectively bound to the CDKs activated transiently by them. Through the activity of CDKs, they catalyze the phosphorylation of different substrates to achieve the promotion and transformation of different phases of the cell cycle. The CDK family includes $1-13$, cyclin is divided into $A-L$, and different CDKs are connected to different cyclins. CDK4/6-specific activation is closely related to the proliferation of some tumors. $\mathrm{Rb}$ is present in approximately $80 \%$ of human tumors, and abnormalities in the cyclin D-CDK4/6-INK4-Rb pathway are common [24]. Cyclindependent kinase (CDK) is a type of serine/threonine (Thr) kinase, which is an important signal transduction molecule in cell and CDK-cyclin formed by cyclin Complex, involved in cell growth, proliferation, dormancy, or entering apoptosis. It is characterized by: (1) p16INK4a gene deletion, point mutation, or DNA methylation leading to inactivation of p16INK4a; (2) CDK4 gene amplification or point muta $T$ cells can induce other cells to activate or interfere with lysis. CD3, CD4, and CD8 cells are involved in T cell transcription of activation signals. Toll-Like receptors (TLRs) play an important role in the identification of invading pathogenic microorganisms in early congenital immunity. These evolutionarily preserved receptors are homologous to the Drosophila Toll protein family in structure, recognizing highly conserved structural motifs (Motifs)-pathogen-associated molecular patterns expressed only on pathogenic microorganisms molecular pattern (PAMP). TLRs are stimulated by PAMPs to initiate a signal cascade including some proteins, leading to the activation of the transcription factor NF-kB, inducing the secretion of pro-inflammatory cytokines and effector cytokines directly involved in the adaptive immune response. TTGB2: Integrin B2, CD18, is an important member of the integrin family of adhesion molecules. It binds to different integrin subunits to form the leukocyte adhesion receptor group. It is 
mainly expressed in white blood cells, and its ligands are TCAM, iC3b, and fibrinogen. Its cytoplasmic region is linked to a variety of cytoskeleton proteins and is involved in signal transduction. Its genetic defects lead to leukocyte adhesion deficiency syndrome. ITGB2 mainly exists in natural killer cell mediated cytotoxicity pathway. In this study, the biological macromolecule interaction instrument was used to verify the binding of the target protein TNF with scopolide, which had the highest score in the docking experiment. The binding effect was good. It was confirmed that scopolide could act on TNF and participate in its corresponding functions. It was confirmed that scorolactone had antimalarial effect. However, at the same time, the binding rates of artemisinin, artemisinin B, artemisinic acid and TNF were very low, which may be because the three compounds do not act on TNF. It also showed that the mechanism of antimalarial effect of artemisinin may be different. The combination of the four ingredients with TNF was very high, indicating that the four ingredients have a synergistic effect. The combined use of the four ingredients can reduce the amount of artemisinin and scopolamine used but achieve the same antimalarial effect. The characteristics of multi-component, multi-target, and synergistic effects of traditional Chinese medicine preparations. Many experiments have proved that TNF has a certain killing effect on Plasmodium. TNF must be assisted by certain factors (or factors) in the body in order to exert its ability to damage Plasmodium, which means TNF is not a terminal effector that directly kills Plasmodium. At present, it is speculated that the immune-protective mechanism of TNF may have the following types: (1) enhance the phagocytosis function of phagocytic cells: the study found that neutrophils were treated with different doses of TNF for 30 minutes and were bred with Plasmodium falciparum, and the neutrophils were found. The phagocytosis of each stage of Plasmodium was strengthened, and the extent of the increase was positively correlated with the dose of TNF within a certain range; (2)Via reactive oxygen mediator: when TNF and macrophages were co-incubated for 30 min, the release of reactive oxygen species (ROS from macrophages) was detected, and this ROS can kill Plasmodi.

\section{Conclusion}

Based on existing literature, data mining methods were used to find the targets of the antimalarial active ingredients, artemisinin and scopolamine in Artemisia annua, including 20 proteins related to artemisinin's antimalarial(IL-6, ACHE, PC3, IPOB , CYC, TNF-a, UGT1A9, CASP3, XDH, IL-1 $\beta$, VEGF, CAT, CREB, AMPK, UGT1A6, ADR, MAPK, COX2, LB24AB, CYP450), and 19 scopolamine antimalarial related proteins(TNF-a, PI3K, IL-8, IL-6, VEGF, IL-1 $\beta$, MAPK, CD4, SP2, CTNNB, CASP3, PR01400, IgE, IL-4, ICAM1, P38, STAT3, TLR4, API4), artemisinic acid and proteins not related to artemisinin.

The combination of the four components of artemisinin, artemisinin B, artemisinic acid, and stigmalactone has good binding to TNF.

\section{Abbreviations}

NHS: N-hydroxysuccinimide; DMSO: dimethyl sulfoxide; PPI: protein-protein interaction; KEGG: kyoto encyclopediaof genesand genomes; Thr: threonine; CDK: cyclin-dependent kinase; PAMP: pathogenic 
microorganisms molecular pattern.

\section{Declarations}

\section{Acknowledgements}

We thank Dr. Zhenyu Zhao for his critical reading and the key project at central government level for providing the support to the case investigation.

\section{Authors' contributions}

Z-yP, W-L, H-IQ integrating pharmacokinetics study, Z-xB, X-hY, network analysis, and experimental validation to research the antimalaria mechanism of Artemisinin, Scopoletin, Arteannuin B and Artemisic acid. Z-yP and H-IQ write the manuscript.

\section{Funding}

This work was funded by Key project at central government level: The ability establishment of sustainable use for valuable Chinese medicine resources, grant No. [2060302].

\section{Availability of data and materials}

All relevant data are included in this report.

\section{Ethics approval and consent to participate}

There are no ethics statement in this paper.

\section{Consent}

Not applicable.

\section{Competing interests}

The authors declare that they have no competing interests.

\section{Author details}

1. China Academy of Chinese Medical Sciences, Beijing 100700, China; 2. State Key Laboratory Breeding Base of Dao-di Herbs, National Resource Center for Chinese Materia Medica, China Academy of Chinese Medical Sciences, Beijing 100700, China;3. Institute of Chinese Materia Medica, China Academy of Chinese Medical Sciences, Beijing 100700, China.

\section{References}


1. Organization WH: World malaria report 2015. World Health Organization; 2016.

2. Sá JM, Twu O, Hayton K, Reyes S, Fay MP, Ringwald P, Wellems TE: Geographic patterns of Plasmodium falciparum drug resistance distinguished by differential responses to amodiaquine and chloroquine. Proceedings of the National Academy of Sciences 2009, 106:18883-18889.

3. Hsiang MS, Hwang J, Tao AR, Liu Y, Bennett A, Shanks GD, Cao J, Kachur SP, Feachem RG, Gosling RD: Mass drug administration for the control and elimination of Plasmodium vivax malaria: an ecological study from Jiangsu province, China. Malaria journal 2013, 12:383.

4. Zhang L, Zhou S, Feng J, Fang W, Xia Z: Malaria situation in the People's Republic of China in 2014. Chin J Parasitol Parasit Dis 2015, 33:319-326.

5. Mclntosh H, Olliaro P: Artemisinin derivatives for treating severe malaria. Cochrane Database of Systematic Reviews 1998.

6. Jambou R, Legrand E, Niang M, Khim N, Lim P, Volney B, Ekala MT, Bouchier C, Esterre P, Fandeur T: Resistance of Plasmodium falciparum field isolates to in-vitro artemether and point mutations of the SERCA-type PfATPase6. The Lancet 2005, 366:1960-1963.

7. Vijaykadga S, Rojanawatsirivej C, Cholpol S, Phoungmanee D, Nakavej A, Wongsrichanalai C: In vivo sensitivity monitoring of mefloquine monotherapy and artesunate-mefloquine combinations for the treatment of uncomplicated falciparum malaria in Thailand in 2003. Tropical Medicine \& International Health 2006, 11:211-219.

8. Noedl H, Se Y, Schaecher K, Smith BL, Socheat D, Fukuda MM: Evidence of artemisinin-resistant malaria in western Cambodia. New England Journal of Medicine 2008, 359:2619-2620.

9. W Muregi F, G Kirira P, Ishih A: Novel rational drug design strategies with potential to revolutionize malaria chemotherapy. Current medicinal chemistry 2011, 18:113-143.

10. Gil JP, Krishna S: pfmdr1 (Plasmodium falciparum multidrug drug resistance gene 1): a pivotal factor in malaria resistance to artemisinin combination therapies. Expert review of anti-infective therapy 2017, 15:527-543.

11. Wang J, Xu C, Liao FL, Jiang T, Krishna S, Tu Y: A Temporizing Solution to "Artemisinin Resistance". New England Journal of Medicine 2019, 380:2087-2089.

12. Hopkins AL: Network pharmacology. Nature biotechnology 2007, 25:1110.

13. Paolini GV, Shapland RH, van Hoorn WP, Mason JS, Hopkins AL: Global mapping of pharmacological space. Nature biotechnology 2006, 24:805.

14. Morris GM, Lim-Wilby M: Molecular docking. In Molecular modeling of proteins. Springer; 2008: 365382

15. Wishart DS, Knox C, Guo AC, Cheng D, Shrivastava S, Tzur D, Gautam B, Hassanali M: DrugBank: a knowledgebase for drugs, drug actions and drug targets. Nucleic acids research 2007, 36:D901D906.

16. Chen JY, Mamidipalli S, Huan T: HAPPI: an online database of comprehensive human annotated and predicted protein interactions. BMC genomics 2009, 10:S16. 
17. Nair M, Basile D: Bioconversion of arteannuin B to artemisinin. Journal of natural products 1993 , 56:1559-1566.

18. Wu W, Yuan M, Zhang Q, Zhu Y, Yong L, Wang W, Qi Y, Guo D: Chemotype-dependent metabolic response to methyl jasmonate elicitation in Artemisia annua. Planta medica 2011, 77:1048-1053.

19. Lévesque F, Seeberger PH: Continuous-flow synthesis of the anti-malaria drug artemisinin. Angewandte Chemie International Edition 2012, 51:1706-1709.

20. Yang H, Li X: Chemical proteomics and discovery of drug targets. Yao xue xue bao= Acta pharmaceutica Sinica 2011, 46:877-882.

21. Gasteiger J: The scope of chemoinformatics. Handbook of Chemoinformatics: From Data to Knowledge in 4 Volumes 2003:3-5.

22. Koutsoukas A, Simms B, Kirchmair J, Bond PJ, Whitmore AV, Zimmer S, Young MP, Jenkins JL, Glick $\mathrm{M}$, Glen RC: From in silico target prediction to multi-target drug design: current databases, methods and applications. Journal of proteomics 2011, 74:2554-2574.

23. Zheng M, Liu X, Xu Y, Li H, Luo C, Jiang H: Computational methods for drug design and discovery: focus on China. Trends in pharmacological sciences 2013, 34:549-559.

24. Malumbres $M$, Barbacid M: Milestones in cell division: to cycle or not to cycle: a critical decision in cancer. Nature Reviews Cancer 2001, 1:222.

\section{Figures}




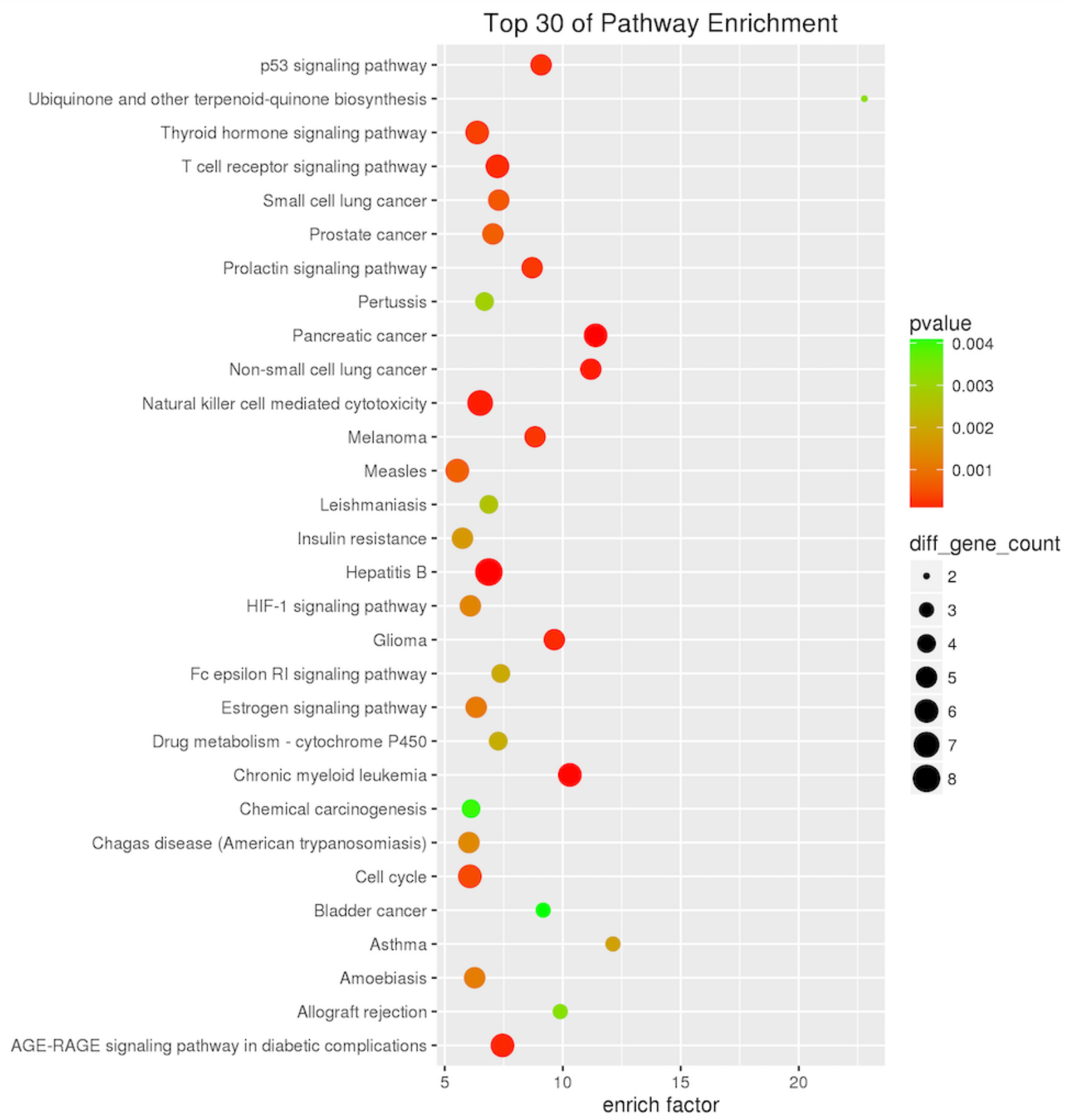

\section{Figure 1}

The above candidate targets significantly participate in multiple immune-related pathways, such as Fc epsilon RI signaling pathway, T cell receptor signaling pathway, and Natural killer cell mediated cytotoxicity; and multiple infectious disease-related pathways, such as Hepatitis B (hepatitis B), Leishmaniasis (leish (Mann disease), Pertussis, Amoebiasis, Measles, Toxoplasmosis and Hepatitis C. 


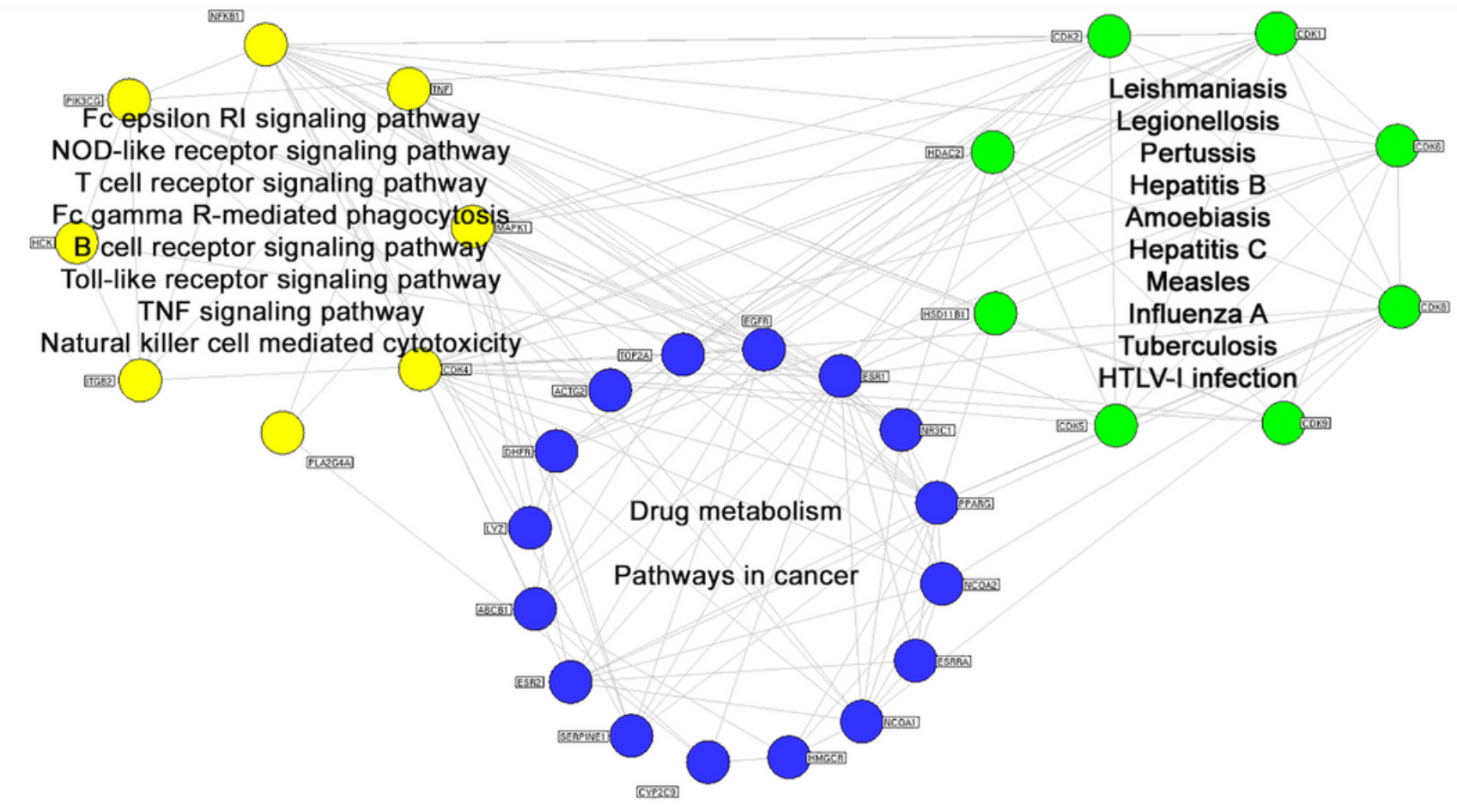

Figure 2

Establishment of an interaction network between Artemisia annua candidate target genes and known antimalarial drug target genes, consisting of 85 nodes and 298 pairs of interactions. 


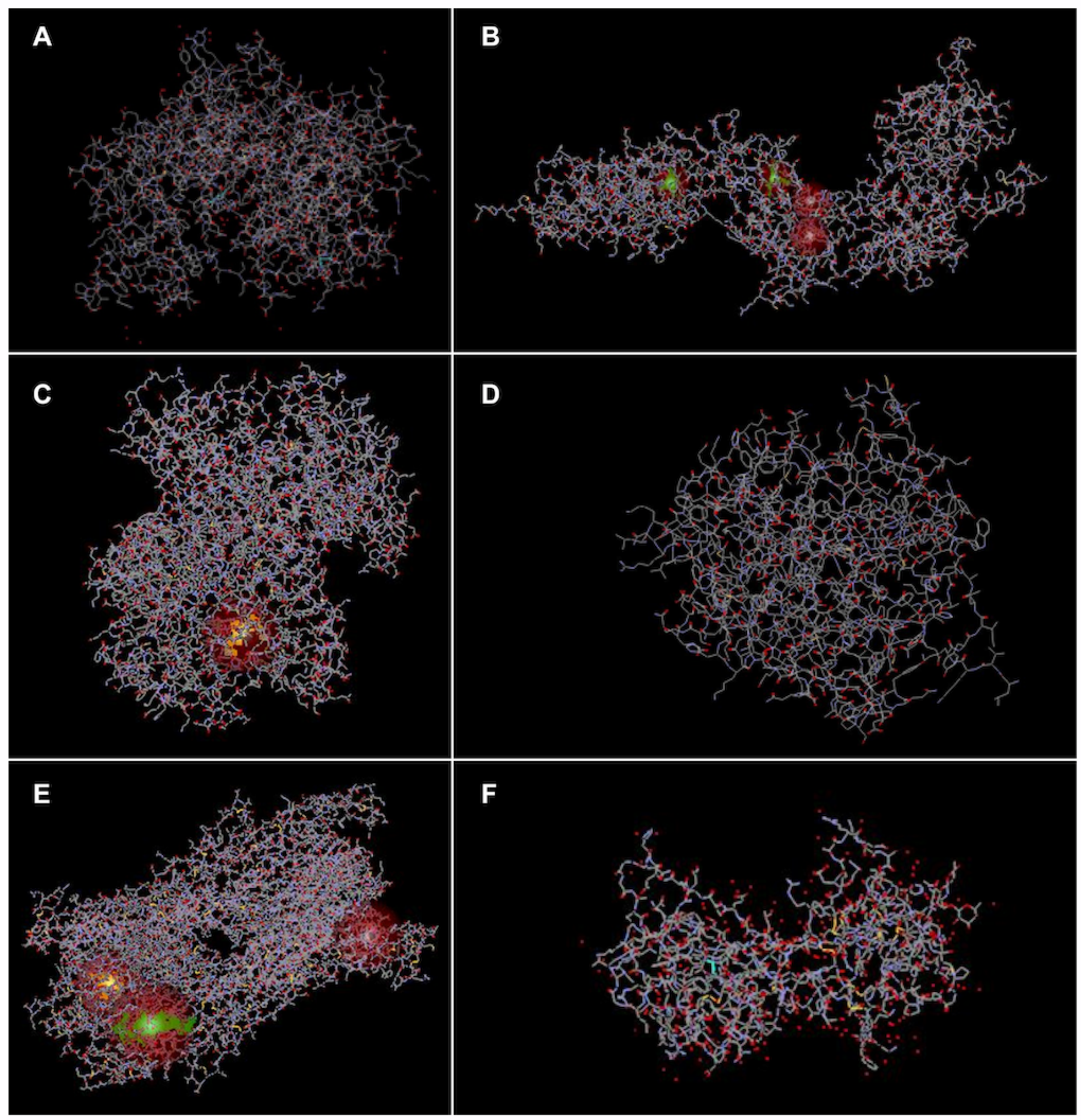

\section{Figure 3}

Molecular Docking by Discovery Studio. A Scopolide with CDK4; B Scopolide with NFKB1; C Scopolide with PIK3CG; D Scopolide with MAPK1; E Scopolide with TNF; F r Artemisinic acid with ITGB2 

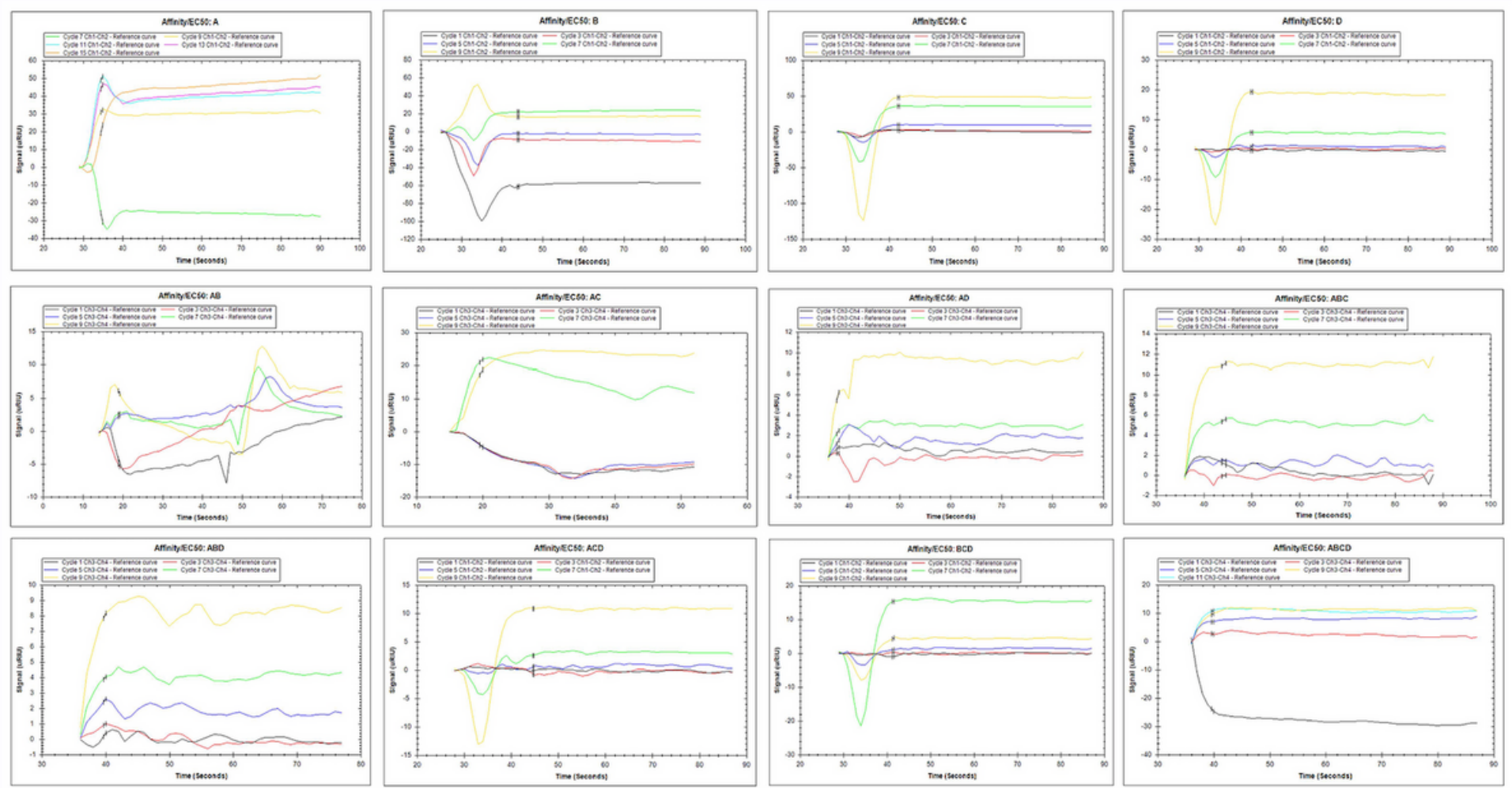

Figure 4

The dissociation constant $(\mathrm{Kd})$ of each group. Grouping is the same as in Table 1. 